\title{
Algorithme de sérologie à deux volets modifiée pour le sérodiagnostic de la maladie de Lyme : contexte canadien
}

Todd Hatchette ${ }^{1 \star}$, Robbin Lindsay ${ }^{2}$ au nom du Groupe de travail sur le diagnostic de la maladie de Lyme

\section{Résumé}

Contexte : La maladie de Lyme $(\mathrm{ML})$ est en progression dans de nombreuses régions du centre et de l'est du Canada. Le sérodiagnostic est la méthode la plus couramment utilisée pour étayer le diagnostic en laboratoire de la ML. La sérologie à deux volets standard (SDVS) de la $\mathrm{ML}$ consiste à détecter les anticorps dirigés contre Borrelia burgdorferi au moyen d'un essai immunoenzymatique (EIA) suivi d'un test d'immuno-empreinte des lgM et/ou des lgG. Une meilleure sensibilité a cependant été observée avec une approche de sérologie à deux volets modifiée (SDVM), qui consiste à remplacer le test d'immuno-empreinte habituel par un second EIA. Cet article résume les données probantes étayant l'utilisation de la SDVM par rapport à la SDVS pour le sérodiagnostic de la ML au Canada.

Méthodes : La sensibilité et la spécificité de différents EIA ont été comparées entre la SDVM et la SDVS chez des patients ayant des antécédents cliniques de ML et vivant dans des régions où la ML est endémique ou à partir d'échantillons issus de la banque de sérums pour la ML. Cette comparaison à été effectuée par un groupe d'experts canadiens en diagnostic de la maladie de Lyme à partir de publications évaluées par les pairs.

Résultats : La SDVM a systématiquement démontré une meilleure sensibilité dans la détection des infections à $B$. burgdorferi au stade précoce par rapport à la SDVS tout en maintenant une spécificité élevée.

Conclusion : Des améliorations dans la sensibilité du diagnostic de la ML sans perte importante de spécificité ont été systématiquement observées lorsque la SDVM était comparée à la SDVS dans les études menées dans les régions où la $M L$ est fortement endémique. Notre groupe de travail souscrit à la recommandation des Centres pour le contrôle et la prévention des maladies (CDC) des États-Unis selon laquelle le sérodiagnostic de la ML par SDVM constitue une solution de substitution acceptable à la STDS. Cette recommandation est conditionnelle à la conception et à la réalisation d'études de validation approfondies sur l'efficacité de la STDM par rapport à la STDS dans le contexte canadien, notamment avec l'évaluation de l'efficacité du test dans les régions où la $\mathrm{ML}$ est faiblement endémique.
Cette oeuvre est mise à la disposition selon les termes de la licence internationale Creative Commons Attribution 4.0

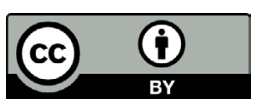

Affiliations

${ }^{1}$ Département de pathologie et de médecine de laboratoire, Université Dalhousie, Halifax NS

${ }^{2}$ Laboratoire national de microbiologie, Agence de la santé publique du Canada, Winnipeg, MB

\section{*Correspondance :}

todd.hatchette@nshealth.ca

Citation proposée : Hatchette TF, Lindsay LR au nom du Groupe de travail sur le diagnostic de la maladie de Lyme. Algorithme de sérologie à deux volets modifiée pour le sérodiagnostic de la maladie de Lyme : contexte canadien. Relevé des maladies transmissibles au Canada 2020;46(5):140-7.

https://doi.org/10.14745/ccdr.v46i05a05f

Mots-clés : Borrelia burgdorferi, maladie de Lyme, sérologie, sérologie à deux volets standard, essais immunoenzymatiques, tests d'immuno-empreinte, diagnostics

\section{Introduction}

La maladie de Lyme (ML) est une infection émergente causée par des spirochètes appartenant au complexe d'espèces Borrelia burgdorferi sensu lato, et transmise à l'homme par des tiques infectées (1). Ses principaux vecteurs sont la tique à pattes noires (Ixodes scapularis) dans l'est et le centre du Canada et la tique occidentale à pattes noires (Ixodes pacificus) en Colombie-Britannique (2). Au Canada, les populations de tiques à pattes noires infectées sont endémiques dans certaines 
parties de la Colombie-Britannique, du Manitoba, de l'Ontario, du Québec, du Nouveau-Brunswick et de la Nouvelle-Écosse (3). Depuis que la $\mathrm{ML}$ est devenue une maladie à déclaration obligatoire à l'échelle nationale, le nombre de Canadiens atteints a augmenté, passant de 144 cas en 2009 à 2025 en 2017 , un chiffre sans doute inférieur à la réalité $(1,2,4)$. Puisque le périmètre géographique des tiques à pattes noires continue de s'étendre, de plus en plus de Canadiens seront susceptibles de contracter la ML (5). On estime à plus de 300000 le nombre de cas de ML aux États-Unis chaque année (6). Le nombre de tests de diagnostic de la ML réalisés aux États-Unis est largement supérieur à celui du Canada (7). Cela a contribué à stimuler les efforts visant à renforcer l'efficacité du diagnostic de la ML, notamment avec l'élaboration et l'approbation de la sérologie à deux volets modifiée (SDVM) (8). Ce document a pour objectif de résumer les données probantes étayant l'efficacité accrue de la SDVM par rapport à l'algorithme d'analyse actuellement utilisé pour diagnostiquer la ML.

\section{Intervention}

Le sérodiagnostic est la méthode de référence la plus souvent utilisée dans le diagnostic en laboratoire de la ML. II consiste à détecter les anticorps dirigés contre $B$. burgdorferi par une sérologie à deux volets standard (SDVS) : un essai immunoenzymatique (EIA) suivi d'un test complémentaire d'immuno-empreinte des lgM et/ou des lgG (figure 1). La plupart des laboratoires de santé publique ou hospitaliers effectuent I'EIA à l'échelle locale tandis que le test d'immuno-empreinte est effectué de manière indépendante par les laboratoires de santé publique provinciaux de la Colombie-Britannique et de I'Ontario (et bientôt du Québec) ou par le Laboratoire national de microbiologie (LNM). Le LNM effectue le test d'immuno-empreinte pour toutes les provinces lorsqu'on soupçonne la présence de la $\mathrm{ML}$ chez des patients qui ont voyagé en dehors de l'Amérique du Nord (figure 1). Quel que soit le type d'analyse, les résultats sont examinés par le personnel de laboratoire et communiqués au médecin demandeur; les résultats positifs sont également transmis aux autorités locales/provinciales de santé publique.

Il existe différents EIA pour le premier volet de la SDVS, notamment ceux composés de sonicats de cellules entières (SCE) de la souche de laboratoire de B. burgdorferi B31. Plus récemment, des EIA fondés sur des peptides synthétiques contenant des régions conservées parmi plusieurs souches de $B$. burgdorferi, comme la séquence protéique variable majeure exprimée (VIsE) de la lipoprotéine de surface, le peptide C6 (région invariable 6 de la protéine VIsE) ou le peptide C10 (partie $\mathrm{N}$-terminal conservée de la protéine de surface externe $\mathrm{C}$ ), ont été mis au point $(8,9)$. Bien que les essais les plus récents présentent une spécificité supérieure à celle des essais à base de SCE, ils ne sont toujours pas assez spécifiques pour être utilisés comme seul essai. Il est donc recommandé d'effectuer une analyse complémentaire par test d'immuno-empreinte (9-12). La SDVS est soumis à un certain nombre de contraintes techniques, notamment le fait que les tests d'immuno-empreinte sont plus difficiles à réaliser que les EIA et que l'interprétation des résultats de l'immuno-empreinte peut être subjective, ce qui peut induire une variabilité inter- et intra-laboratoire (11). Par ailleurs, comme les tests d'immuno-empreinte sont effectués par un nombre relativement faible de laboratoires de diagnostic de référence aux États-Unis (7) et au Canada, les délais d'exécution sont généralement plus longs que pour les $\operatorname{EIA}(8,11)$.

\section{Figure 1 : Schéma illustrant les étapes de la sérologie à deux volets standard et de la sérologie à deux volets modifiée de la maladie de Lyme}

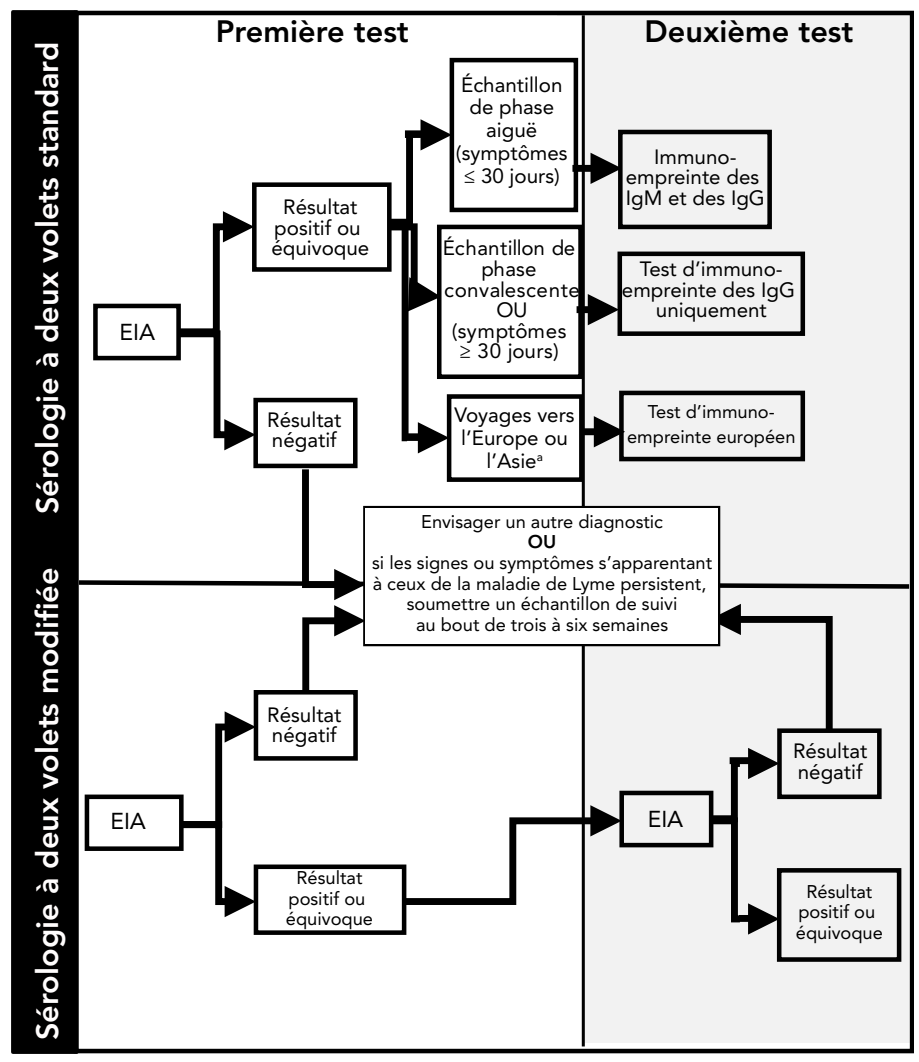

Abréviations : EIA, essai immunoenzymatique; lgG, immunoglobuline G; IgM, immunoglobuline $M$

a Il convient de noter que les cas suspects de ML originaires d'Europe ou d'Asie continuent d'être analysés par la sérologie à deux volets standard

L'efficacité de l'algorithme de la SDVS dépend également du stade de l'infection. Une revue systématique récente a montré que la sensibilité de la SDVS pour la ML était faible au stade localisé précoce de l'infection (moins de $50 \%$ ), mais particulièrement élevée aux stades tardifs de l'infection (proche de $100 \%$ ) (13). De ce fait, le diagnostic et le traitement de la $\mathrm{ML}$ au stade localisé précoce reposent uniquement sur les symptômes cliniques des patients ayant été exposés dans des régions où la tique à pattes noires est endémique (10). Le diagnostic de la $\mathrm{ML}$ au stade précoce peut néanmoins s'avérer difficile, car certains patients atteints d'une infection à $B$. burgdorferi au stade localisé précoce ne présentent pas d'érythème migrant et peuvent manifester des symptômes qui se confondent avec ceux d'autres maladies $(9,14)$. Il est donc important d'améliorer la sensibilité du test diagnostique des 
infections au stade localisé précoce pour recenser les patients atteints de la $\mathrm{ML}$, les traiter le plus rapidement possible et, éventuellement, empêcher la dissémination de l'infection et son évolution vers une forme grave de la maladie.

\section{Résultats}

\section{Sérologie à deux volets modifiée pour le sérodiagnostic de la maladie de Lyme}

Plusieurs études ont évalué l'utilisation d'une approche de SDVM dans laquelle le test d'immuno-empreinte habituel est remplacé par un second EIA (figure 1). De multiples combinaisons différentes d'EIA ont été utilisées dans cette " approche à double EIA ", notamment un EIA à base de SCE suivi d'un EIA basé sur le peptide $\mathrm{C6}$, un EIA basé sur la protéine VIsE suivi d'un EIA basé sur le peptide C6, un EIA basé sur le peptide C6 suivi d'un EIA basé sur la protéine VIsE et un EIA basé sur la protéine VIsE et le peptide $\mathrm{C} 10$ suivi d'un EIA à base de SCE (15-20). Les échantillons utilisés dans le cadre de ces études provenaient de petites cohortes de patients atteints d'une forme aiguë de la $M L(15,18)$, ou des comparaisons étaient réalisées au moyen d'échantillons bien caractérisés issus de la banque de sérums pour la ML des Centres pour le contrôle et la prévention des maladies (CDC) $(16,19,21)$. Les études portaient sur des échantillons d'enfants $(17,20)$ et d'adultes $(16,19,21)$. À quelques exceptions près (22), ces études ont été menées uniquement sur des patients originaires des États-Unis. La SDVM n'a pas été pleinement validée en vue de son utilisation auprès de patients ayant été exposés en Europe ou en Asie.

Bien que différentes combinaisons d'EIA aient été utilisées dans les algorithmes de la SDVM, la STDM a systématiquement démontré une meilleure sensibilité que la SDVS pour la détection des infections à $B$. burgdorferi, en particulier la $M L$ au stade localisé précoce. Fait important, la SDVM présentait une sensibilité équivalente dans la détection des infections au stade tardif et une spécificité comparable à celle de la SDVS, et ce, quelle que soit la combinaison d'EIA utilisée pour la SDVM (se reporter aux tableaux 1 et 2). Plus récemment, le Secrétariat américain aux produits alimentaires et pharmaceutiques (FDA) a validé un algorithme de SDVM pour la confirmation en laboratoire de la maladie de Lyme chez les patients ayant acquis

\section{Tableau 1 : Sensibilité de la sérologie à deux volets modifiée et de la sérologie à deux volets standard de la maladie de Lyme}

\begin{tabular}{|c|c|c|c|c|c|}
\hline $\begin{array}{c}\text { Taille de } \\
\text { l'échantillon }\end{array}$ & Référence & Manifestations cliniques & Combinaisons d'EIA utilisées ${ }^{a}$ & $\begin{array}{c}\text { Sensibilité de la } \\
\text { SDVM \% } \\
\text { (IC ou plage) }\end{array}$ & $\begin{array}{c}\text { Sensibilité de la } \\
\text { SDVS \% } \\
\text { (IC ou plage) }^{b}\end{array}$ \\
\hline 140 & (15) & $\mathrm{EM}, \mathrm{NBP}, \mathrm{CL}$ & SCE suivi de C6 & 61 (IC 53-69) & 48 (IC 40-56) \\
\hline 318 & (11) & EM, NBP & SCE suivi de C6 & 60 (IC 55-66) & 41 (IC 36-46) \\
\hline 55 & (18) & EM de phase aiguë & $\begin{array}{l}\text { SCE suivi de C6; SCE suivi de VlsE CFLIA; VlsE } \\
\text { FLIA suivi de C6 }\end{array}$ & $\begin{array}{r}42,7 \\
(38,0-54,0) \\
\end{array}$ & $32(25-36)$ \\
\hline 47 & (18) & EM de phase convalescente & $\begin{array}{l}\text { SCE suivi de C6; SCE suivi de VIsE CFLIA; VIsE } \\
\text { FLIA suivi de C6 }\end{array}$ & $\begin{array}{r}70 \\
(66-72)\end{array}$ & $57,3(55,0-60,0)$ \\
\hline 95 & (16) & EM, NBP, CL & Vidas suivi de $\mathrm{C} 6$ or $\mathrm{Vls}^{\mathrm{c}}$ & $\begin{array}{r}66,8 \\
(65,2-68,4)\end{array}$ & $60,2(56,8-64,2)$ \\
\hline 114 & (17) & $\begin{array}{l}\text { Tous stades de la maladie } \\
\text { combinés }\end{array}$ & SCE suivi de C6 & $\begin{array}{r}79,8 \\
(\text { IC } 71,1-86,5)\end{array}$ & 81,6 (IC 73,0-88,0) \\
\hline 40 & (19) & EM de phase aiguë & $\begin{array}{l}\text { VlsE suivi de C6; SCE suivi de C6; SCE suivi de } \\
\text { VlsE }\end{array}$ & $\begin{array}{r}54,3 \\
(50,0-58,0) \\
\end{array}$ & $45,3(43,0-50,0)$ \\
\hline 38 & (19) & EM de phase convalescente & $\begin{array}{l}\text { VlsE suivi de C6; SCE suivi de C6; SCE suivi de } \\
\text { VlsE }\end{array}$ & 77 (76-79) & $61 ; 61 ; 63$ \\
\hline 124 & (19) & $\begin{array}{l}\text { Tous stades de l'infection } \\
\text { combinés }\end{array}$ & $\begin{array}{l}\text { VlsE suivi de C6; SCE suivi de C6; SCE suivi de } \\
\text { VlsE }\end{array}$ & $\begin{array}{r}76,7 \\
(75,0-78,0) \\
\end{array}$ & $66 ; 67 ; 71$ \\
\hline 30 & (25) & EM de phase aiguë & VlsE/pepC10 suivi de SCE & 73,3 & 50 \\
\hline 30 & (25) & EM de phase convalescente & VlsE/pepC10 suivi de SCE & 83,3 & 76,7 \\
\hline 56 & (25) & $\begin{array}{l}\text { Infection au stade disséminé } \\
\text { précoce }^{\text {a }}\end{array}$ & VlsE/pepC10 suivi de SCE & 66,1 & 60,7 \\
\hline 29 & (15) & $\mathrm{AL}, \mathrm{NBT}$ & SCE suivi de C6 & 100 (IC 86-100) & 100 (IC 86-100) \\
\hline 122 & (11) & AL, NBT & SCE suivi de C6 & 98 (IC 93-99) & 96 (IC 91-98) \\
\hline 29 & (16) & $\mathrm{AL}$ & Vidas suivi de $\mathrm{C} 6$ or VlsE & 100 & $98,9(97-100)$ \\
\hline 50 & (25) & $\begin{array}{l}\text { Infection au stade disséminé } \\
\text { tardifc }\end{array}$ & VlsE/pepC10 suivi de SCE & 100 & 100 \\
\hline
\end{tabular}

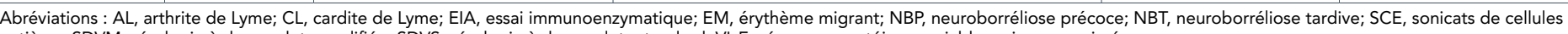
entières; SDVM, sérologie à deux volets modifiée; SDVS, sérologie à deux volets standard; VIsE, séquence protéique variable majeure exprimée

Type d'EIA et ordre dans lequel les EIA ont été réalisés; se reporter aux publications originales pour consulter les renseignements fournis par le fabricant

${ }^{-}$Se reporter aux publications originales pour obtenir des précisions sur l'EIA et le test d'immuno-empreinte utilisés dans les algorithmes de la SDVS

Les données issues de ces deux combinaisons d'ElA ont été regroupées, car il n'existe aucune différence significative entre elles 
Tableau 2 : Spécificité de la sérologie à deux volets modifiée et de la sérologie à deux volets standard de la maladie de Lyme

\begin{tabular}{|c|c|c|c|c|c|}
\hline $\begin{array}{l}\text { Taille de } \\
\text { l'échantillon }\end{array}$ & Référence & Cohorte de patients ${ }^{a}$ & $\begin{array}{c}\text { Combinaisons d'EIA } \\
\text { utilisées }^{b}\end{array}$ & $\begin{array}{c}\text { Sensibilité de la } \\
\text { SDVM \% } \\
\text { (IC ou plage) }\end{array}$ & $\begin{array}{l}\text { Sensibilité de la } \\
\text { SDVS \% } \\
\text { (IC ou plage) }^{c}\end{array}$ \\
\hline \multicolumn{6}{|l|}{ Témoins totaux } \\
\hline 1300 & $(15)$ & $\begin{array}{l}\text { Témoins en bonne santé et } \\
\text { symptomatiques }\end{array}$ & SCE suivi de C6 & $\begin{array}{r}99,5 \\
(I C 98,9-99,8)\end{array}$ & $\begin{array}{r}99,5 \\
\text { (IC 98,9-99,8) }\end{array}$ \\
\hline 2208 & $(11)$ & $\begin{array}{l}\text { Témoins en bonne santé et patients } \\
\text { atteints d'autres maladies }\end{array}$ & SCE suivi de C6 & $\begin{array}{r}99,5 \\
(I C 99,1-99,8) \\
\end{array}$ & $\begin{array}{r}99,5 \\
(\text { IC 99,1-99,7) }\end{array}$ \\
\hline 347 & $(16)$ & $\begin{array}{l}\text { Témoins en bonne santé et patients } \\
\text { atteints d'autres maladies }\end{array}$ & Vidas suivi de $\mathrm{C} 6$ ou $\mathrm{VlsE}^{c}$ & (IC 96,2-99,3) & $\begin{array}{r}98,3 \\
(\text { IC } 96,2-99,3)\end{array}$ \\
\hline 931 & $(17)$ & $\begin{array}{l}\text { Témoins en bonne santé et } \\
\text { symptomatiques }\end{array}$ & SCE suivi de C6 & $\begin{array}{r}96,6 \\
(94,6-97,6)\end{array}$ & $\begin{array}{r}98,7 \\
(96,6-100)\end{array}$ \\
\hline 347 & $(19)$ & $\begin{array}{l}\text { Témoins en bonne santé et patients } \\
\text { atteints d'autres maladies }\end{array}$ & $\begin{array}{l}\text { VlsE suivi de C6; SCE suivi } \\
\text { de C6; SCE suivi de VlsE }\end{array}$ & $\begin{array}{r}98,6 \\
(97,7-99,4)\end{array}$ & $\begin{array}{r}98,1 \\
(95,7-99,7)\end{array}$ \\
\hline 190 & $(25)$ & $\begin{array}{l}\text { Témoins en bonne santé et patients } \\
\text { atteints d'autres maladies }\end{array}$ & VlsE/C10 suivi de SCE & $\begin{array}{r}98,9 \\
(97,8-100)\end{array}$ & 100 \\
\hline \multicolumn{6}{|c|}{ Témoins en mauvaise santé } \\
\hline 54 & $(15)$ & Témoins symptomatiques & SCE suivi de C6 & 100 & 100 \\
\hline 50 & $(18)$ & Patients atteints d'autres maladies & $\begin{array}{l}\text { SCE suivi de C6; SCE suivi } \\
\text { de VIsE CLIA; VIsE CLIA } \\
\text { suivi de C6 }\end{array}$ & $\begin{array}{r}99,3 \\
(98-100)\end{array}$ & 100 \\
\hline 144 & $(16)$ & Patients atteints d'autres maladies & Vidas suivi de $\mathrm{C} 6$ ou VlsE ${ }^{d}$ & $\begin{array}{r}98,2 \\
(96,5-100)\end{array}$ & $\begin{array}{r}97,1 \\
(94,4-99,3)\end{array}$ \\
\hline 830 & $(17)$ & Témoins symptomatiques & SCE suivi de C6 & $\begin{array}{r}96,5 \\
(94,6-97,6)\end{array}$ & $\begin{array}{r}98,7 \\
(96,6-100)\end{array}$ \\
\hline 144 & $(19)$ & Patients atteints d'autres maladies & $\begin{array}{l}\text { VlsE suivi de C6; SCE suivi } \\
\text { de C6; SCE suivi de VlsE }\end{array}$ & $\begin{array}{r}98,1 \\
(96,5-100) \\
\end{array}$ & $\begin{array}{r}97,4 \\
(95,7-99,7) \\
\end{array}$ \\
\hline 90 & $(25)$ & Patients atteints d'autres maladies & VlsE/C10 suivi de SCE & 97,8 & 100 \\
\hline \multicolumn{6}{|c|}{$\begin{array}{l}\text { Abréviations : EIA, essai immunoenzymatique; SCE, sonicats de cellules entières; SDVM, sérologie à deux volets modifiée; SDVS, sérologie à deux volets standard; VIsE, séquence protéique variable } \\
\text { majeure exprimée } \\
\text { a Contrairement aux témoins en bonne santé, les témoins symptomatiques présentaient des symptômes cliniques qui s'apparentaient à ceux de la maladie de Lyme (ML), mais ne correspondaient pas } \\
\text { à la définition de cas de la ML établie par les auteurs; se reporter aux publications originales pour obtenir la liste des autres maladies semblables (comme la syphilis, la fibromyalgie et la sclérose en } \\
\text { plaques) } \\
\text { b Type d'EIA et ordre dans lequel les EIA ont été réalisés pour la SDVM; se reporter aux publications originales pour consulter les renseignements fournis par le fabricant } \\
\text { c Se reporter aux publications originales pour obtenir des précisions sur l'EIA et le test d'immuno-empreinte utilisés dans les algorithmes de SDVS } \\
\text { d Les données issues de ces deux combinaisons d'EIA ont été regroupées, car il n'existe aucune différence significative entre elles }\end{array}$} \\
\hline
\end{tabular}

leur infection en Amérique du Nord (23). Cet algorithme de sérodiagnostic a été validé par les CDC des États-Unis, qui le considèrent comme une solution de substitution acceptable à la SDVS, car « l'autorisation de nouveaux tests sérologiques de la maladie de Lyme par la FDA signifie que l'efficacité du test a été évaluée et que celle-ci est substantiellement équivalente ou supérieure à celles des tests prédicats commercialisés légalement » (24). On ignore si l'approche de la SDVM sera validée aux États-Unis pour les patients susceptibles d'avoir contracté la ML en dehors de l'Amérique du Nord. Au Canada, néanmoins, l'algorithme de la SDVS continuera d'être utilisé en effectuant des tests européens auprès des Canadiens soupçonnés d'avoir contracté la ML en dehors de l'Amérique du Nord (figure 1).

\section{Forces et faiblesses de la sérologie à deux volets modifiée}

Outre une meilleure sensibilité pour la détection des infections à $B$. burgdorferi au stade précoce, l'interprétation des résultats de la SDVM est moins subjective que celle du test d'immunoempreinte (tableau 3). La SDVM s'est également révélée plus rentable que la SDVS (26). Les tests sont moins laborieux et peuvent être effectués à l'aide d'instruments ou de plates-formes automatisés (8). À ce titre, la SDVM ne nécessite pas de procéder à un test spécialisé (immuno-empreinte) dans un laboratoire de référence; elle peut donc être réalisée par n'importe quel laboratoire assurant actuellement un test sérologique. Ces différences peuvent contribuer à réduire les délais d'obtention des résultats $(8,9)$.

Les résultats de la SDVM peuvent être soit positifs, soit négatifs, ce qui permet une interprétation plus " tranchée " qu'avec la SDVS, car les tests d'immuno-empreinte des lgM et des lgG peuvent produire des résultats différents qui peuvent être source de confusion pour les médecins (11). Bien que la SDVM présente une meilleure sensibilité que la SDVS, celle-ci demeure inférieure à $90 \%$. Par conséquent, les patients atteints de la $\mathrm{ML}$ au stade localisé précoce devraient continuer à être traités sur la base de leur tableau clinique plutôt que leurs résultats 
Tableau 3 : Avantages et inconvénients de la sérologie à deux volets modifiée par rapport à la sérologie à deux volets standard de la maladie de Lyme

\begin{tabular}{|c|c|}
\hline Avantages & Inconvénients \\
\hline $\begin{array}{l}\text { - Meilleure sensibilité pour la } \\
\text { détection de l'infection au } \\
\text { stade précoce (plus de } 25 \% \\
\text { d'amélioration) } \\
\text { - Moins coûteux que la SDVS } \\
\text { - Moins laborieux } \\
\text { - Moins subjectif } \\
\text { - Les essais } \\
\text { immunoenzymatiques sont } \\
\text { réalisés à l'échelle locale } \\
\text { plutôt que par un laboratoire } \\
\text { spécialisé, ce qui contribue à } \\
\text { réduire les délais d'exécution } \\
\text { - Des délais d'exécution réduits } \\
\text { permettent de faciliter le } \\
\text { diagnostic (en phase aiguë et } \\
\text { convalescente) de la ML au } \\
\text { stade localisé précoce sans } \\
\text { érythème migrant }\end{array}$ & $\begin{array}{l}\text { - Les patients qui présentent } \\
\text { un érythème migrant devront } \\
\text { malgré tout être traités par } \\
\text { antibiotiques étant donné que } \\
\text { la sensibilité de l'algorithme de } \\
\text { sérodiagnostic est inférieure à } \\
90 \% \\
\text { - Comme c'est le cas pour la } \\
\text { SDVS, il est impossible de } \\
\text { différencier une réinfection } \\
\text { d'une infection ancienne } \\
\text { - L'incidence de la SDVM sur } \\
\text { la spécificité dans les régions } \\
\text { où la prévalence est faible } \\
\text { demeure incertaine } \\
\text { - La SDVS peut être nécessaire } \\
\text { ou bénéfique chez les patients } \\
\text { atteints d'arthrite de Lyme, } \\
\text { compte tenu de la perte } \\
\text { potentielle de spécificité } \\
\text { associée à certains essais } \\
\text { immunoenzymatiques } \\
\text { polyvalents }\end{array}$ \\
\hline
\end{tabular}

Abréviations : ML, maladie de Lyme; SDVM, sérologie à deux volets modifiée; SDVS, sérologie à deux volets standard

sérologiques. La rapidité des délais d'exécution de la SDVM peut néanmoins s'avérer particulièrement utile dans l'évaluation des patients visés par une suspicion clinique de ML, mais sans éruption cutanée (érythème migrant), ou des patients qui présentent des symptômes qui se confondent avec ceux d'autres infections (p. ex. paralysie de Bell ou arthrite), auquel cas les résultats sérologiques faciliteront le diagnostic (8). Dans ses dernières lignes directrices fondées sur des données probantes, le Royaume-Uni formule la recommandation suivante : "Si l'on soupçonne la présence de la ML chez des personnes ayant été dépistées dans les quatre semaines suivant l'apparition des symptômes et chez qui le test ELISA s'est révélé négatif, répéter ce test quatre à six semaines après la date du premier test » (12). À l'heure actuelle, même si l'EIA de phase convalescente se révèle positif, il reste malgré tout nécessaire de procéder à une analyse complémentaire avec un test d'immuno-empreinte dans le cadre de la SDVS. Au vu des meilleurs délais d'exécution associés à la SDVM, les cliniciens pourraient être enclins à suivre la recommandation de l'Institut national pour la santé et l'excellence des soins (NICE) et envisager un test en phase aiguë et convalescente, ce qui permettrait d'accroître la certitude diagnostique du test sérologique auprès des patients qui ne présentent pas d'érythème migrant. Cet aspect est particulièrement important lorsque la suspicion clinique est faible, comme pour les patients sans antécédents connus d'exposition aux tiques dans les régions où le risque de contracter la ML est élevé.

En dépit de ses nombreux avantages, la SDVM présente également des inconvénients. Étant donné que les anticorps dirigés contre $B$. burgdorferi peuvent persister pendant des mois, voire des années après la première infection (27), I'algorithme de la SDVM (tout comme la SDVS) ne peut différencier une infection active d'une ancienne infection, ce qui complique le diagnostic sérologique d'une réinfection à B. burgdorferi. En outre, il est possible que l'algorithme de la SDVM génère des faux positifs en fonction du composant lgM des EIA polyclonaux utilisés, car on sait que des faux positifs au test d'immuno-empreinte des IgM surviennent chez les patients en bonne santé ou ceux qui présentent des symptômes de longue date (28-31). II se peut que la très grande efficacité de la SDVS de la ML au stade tardif soit difficile à reproduire dans le format de la SDVM, en particulier lorsque des EIA polyvalents (contenant des épitopes pour les IgM) sont utilisés, et il est probable que les tests d'immuno-empreinte demeurent nécessaires pour évaluer les cas complexes de $\mathrm{ML}$ (8). Face à ce constat, le recours au test d'immuno-empreinte peut toujours être utile chez les patients qui manifestent les symptômes d'une ML au stade tardif (comme l'arthrite de Lyme) ou ceux soupçonnés de faux positif lorsque leurs résultats sérologiques ne concordent pas avec le tableau clinique. Dans ces conditions, il apparaît raisonnable d'envisager la réalisation d'un test d'immuno-empreinte des lgG, car on observe une forte réponse des anticorps IgG chez les patients atteints de la ML au stade tardif et le test d'immuno-empreinte pourrait permettre d'évaluer la réponse à des protéines Borrelia particulières, ce que certains cliniciens jugent utile $(32,33)$. Enfin, la plupart des études sur l'algorithme de la SDVM ont été menées dans des régions où la $\mathrm{ML}$ est fortement endémique et les analyses sérologiques se sont limitées principalement aux patients adultes. Aucune étude n'a pour le moment été menée sur l'efficacité de la SDVM dans les régions où le risque de contracter la $\mathrm{ML}$ est moindre et chez les populations pédiatriques; ces lacunes dans les connaissances devraient être comblées avec le temps (20).

\section{Discussion}

Le Réseau des laboratoires de santé publique du Canada souscrit à la recommandation des CDC (24) selon laquelle le diagnostic sérologique de la ML basé sur une approche de SDVM (remplacement du test d'immuno-empreinte prévu au deuxième volet par un second EIA) constitue une solution de substitution acceptable à la SDVS. Cette recommandation suppose que I'approche de la SDVM a été validée et a démontré une efficacité comparable à celle de la SDVS dans les régions canadiennes où I'incidence de la ML est élevée, ainsi que dans les territoires où I'incidence est faible. Actuellement, seule la Nouvelle-Écosse dispose de données validant l'approche de la SDVM pour le diagnostic de la ML. Portant sur 447 échantillons recueillis auprès de patients atteints de la ML et originaires de cette province, une SDVM consistant en un EIA à base de SCE de B. burgdorferi suivi d'un ElA basé sur le peptide C6 a permis de détecter $25 \%$ plus de cas d'infection au stade localisé précoce que la SDVS, avec une spécificité de 99,5\% (34). Ces résultats vont dans le sens des données publiées dans les études menées dans les régions des États-Unis où la $\mathrm{ML}$ est fortement endémique 
$(11,15)$ et viennent appuyer le recours à la SDVM dans cette province. Cette étude de validation a toutefois été réalisée dans la province canadienne où l'incidence de la ML est la plus élevée (35). D'autres études de validation de la SDVM devront être menées dans les régions du Canada où l'incidence de la ML est plus faible, car il est essentiel que l'efficacité de la SDVM soit documentée chez les populations où la probabilité d'infection pré-test est plus faible $(15,36)$. Une légère baisse de la spécificité peut faire diminuer la valeur prédictive du test (tableau 4); c'est pourquoi il a été recommandé de ne pas entreprendre d'analyse sérologique de la ML lorsque la probabilité prétest était inférieure à $20 \%$ (37). En raison de la variabilité des souches parmi les populations de $B$. burgdorferi observées au Canada (38), et de l'incidence que cette variabilité pourrait avoir sur les tests de diagnostic de la ML (39), il paraît prudent de s'assurer que la sensibilité accrue de la SDVM démontrée par la littérature sera conservée lorsque l'approche sera appliquée dans les différentes régions du Canada qui abritent les souches diverses et variées de $B$. burgdorferi.

\section{Tableau 4 : Valeurs prédictives estimées de la sérologie à deux volets modifiée en fonction du stade de l'infection et de la prévalence estimée de l'infection à B. burgdorferi ${ }^{\mathrm{a}}$}

\begin{tabular}{|l|r|r|r|r|}
\hline \multirow{2}{*}{$\begin{array}{c}\text { Prévalence } \\
\text { estimée } \\
(\%)\end{array}$} & $\begin{array}{c}\text { Valeur prédictive } \\
\text { positive de la } \\
\text { SDVM (\%) }\end{array}$ & \multicolumn{2}{|c|}{$\begin{array}{c}\text { Valeur prédictive } \\
\text { négative de la SDVM (\%) }\end{array}$} \\
\cline { 2 - 5 } & $\begin{array}{r}\text { ML au } \\
\text { stade } \\
\text { localisé } \\
\text { précoce }\end{array}$ & $\begin{array}{l}\text { ML au } \\
\text { stade } \\
\text { tardifc }\end{array}$ & $\begin{array}{c}\text { ML au } \\
\text { stade } \\
\text { localisé } \\
\text { précoce }\end{array}$ & $\begin{array}{c}\text { ML au stade } \\
\text { tardif }\end{array}$ \\
\hline 5 & 84,8 & 91,3 & 97,6 & 100 \\
\hline 3 & 76,6 & 86 & 98,6 & 100 \\
\hline 1 & 51,7 & 66,8 & 99,5 & 100 \\
\hline 0,1 & 9,6 & 16,6 & 100 & 100 \\
\hline
\end{tabular}

Abréviations : ML, maladie de Lyme; SDVM, sérologie à deux volets modifiée

a Valeurs prédictives positives et négatives calculées à l'aide de l'outil

http://vassarstats.net/clin2.html

b Sensibilité estimée à $53 \%$ sur la base des données de la référence 15; spécificité estimée à

$99,5 \%$ sur la base des données issues des études réalisées en Nouvelle-Écosse (33)

c Sensibilité estimée à $99,3 \%$ sur la base des données de la référence 15; spécificité estimée à

$99,5 \%$ sur la base des données issues des études réalisées en Nouvelle-Écosse (33)

Le Groupe de travail sur le diagnostic de la maladie de Lyme du Réseau des laboratoires de santé publique du Canada collabore avec les laboratoires provinciaux pour élaborer des plans de validation de la SDVM. La validation a pour objectif d'établir l'efficacité de la SDVM dans les régions où l'incidence de la $\mathrm{ML}$ est variable (et où les souches de $B$. burgdorferi sont potentiellement différentes) et de déterminer la combinaison, parmi les EIA disponibles au Canada, qui permettrait d'obtenir les données nécessaires pour que les avantages du nouvel algorithme de la SDVM puissent se matérialiser et que la spécificité du test sérologique de la ML puisse être conservée. Un second rapport sera rendu public une fois que ces études auront été achevées.

\section{Conclusion}

Le Secrétariat américain aux produits alimentaires et pharmaceutiques a récemment validé un algorithme diagnostique de la SDVM pour la sérologie de la $\mathrm{ML}$, et les CDC des États-Unis ont recommandé le recours à cette nouvelle approche, la considérant comme une solution de substitution acceptable à la SDVS. Un nombre croissant de publications scientifiques impliquant des patients originaires des États-Unis mettent en évidence une meilleure sensibilité dans la détection de l'infection à la $M L$ au stade localisé précoce, ainsi que le maintien d'une spécificité élevée lorsque les algorithmes de la SDVM sont comparés à la SDVS. Les dernières données de la Nouvelle-Écosse, générées par l'entremise de la SDVM, conduisent à des conclusions similaires. L'amélioration de la sensibilité de la SDVM et la réduction des délais d'exécution associées à cette nouvelle approche justifient des études plus approfondies sur ce nouvel algorithme de diagnostic de la ML en vue de son éventuel déploiement au Canada.

\section{Déclaration des auteurs}

Le Groupe de travail sur le diagnostic de la maladie de Lyme du Réseau des laboratoires de santé publique du Canada est constitué de T. Hatchette (coprésident), L. R. Lindsay (coprésident), K. Bernat, G. Desnoyers, A. Dibernardo, K. Fonseca, G. German, A. Lang, M. Morshed, R. Needle, S. Patel, K. Thivierge et P. VanCaeseele.

\section{Conflit d'intérêts}

Les auteurs ne déclarent aucun conflit d'intérêts.

\section{Remerciements}

Le Réseau des laboratoires de santé publique du Canada a gracieusement fourni un soutien administratif qui s'est révélé d'une grande utilité lors de l'élaboration de cet énoncé de position.

\section{Financement}

Ce projet n'a fait l'objet d'aucun financement particulier.

\section{References}

1. Ogden NH, Bouchard C, Badcock J, Drebot MA, Elias SP, Hatchette TF, Koffi JK, Leighton PA, Lindsay LR, Lubelczyk $C B$, Peregrine AS, Smith RP, Webster D. What is the real number of Lyme disease cases in Canada? BMC Public Health 2019 Jun;19(1):849. DOI PubMed 
2. Agence de la santé publique du Canada. Surveillance de la maladie de Lyme. Ottawa (ON): ASPC; 2018 (Accédé 18-092019). https://www.canada.ca/fr/sante-publique/services/ maladies/maladie-lyme/surveillance-maladie-lyme.html

3. Ogden NH, Koffi JK, Pelcat Y, Lindsay LR. Risque environnemental pour la maladie de Lyme dans l'est et le centre du Canada : un sommaire d'informations récentes en matière de surveillance. Relevé des maladies transmissibles au Canada 2014;40(5):77-86. DOI

4. Henry B, Roth D, Reilly R, MacDougall L, Mak S, Li M, Muhamad M. How big is the Lyme problem? Using novel methods to estimate the true number of Lyme disease cases in British Columbia residents from 1997 to 2008. Vector Borne Zoonotic Dis 2011;11(7):863-8. DOI PubMed

5. Leighton PA, Koffi JK, Pelcat Y, Lindsay LR, Ogden N. Predicting the speed of tick invasion: an empirical model of range expansion for the Lyme disease vector Ixodes scapularis. J Appl Ecol 2012;49(2):457-64. DOI

6. Kuehn BM. CDC estimates 300,000 US cases of Lyme disease annually. JAMA 2013;310(11):1110. DOI PubMed

7. Hinckley AF, Connally NP, Meek JI, Johnson BJ, Kemperman MM, Feldman KA, White JL, Mead PS. Lyme disease testing by large commercial laboratories in the United States. Clin Infect Dis 2014;59(5):676-81. DOI PubMed

8. Marques AR. Revisiting the Lyme disease serodiagnostic algorithm: the momentum gathers. J Clin Microbiol 2018;56(8):e00749-18. DOl PubMed

9. Moore A, Nelson C, Molins C, Mead P, Schriefer M. Current guidelines, common clinical pitfalls, and future directions for laboratory diagnosis of Lyme Disease, United States. Emerg Infect Dis 2016;22(7):1169-77. [Epub ahead of print]. DOI PubMed

10. Wormser GP, Dattwyler RJ, Shapiro ED, Halperin JJ, Steere AC, Klempner MS, Krause PJ, Bakken JS, Strle F, Stanek G, Bockenstedt L, Fish D, Dumler JS, Nadelman RB. The clinical assessment, treatment, and prevention of lyme disease, human granulocytic anaplasmosis, and babesiosis: clinical practice guidelines by the Infectious Diseases Society of America. Clin Infect Dis 2006;43(9):1089-134.

DOI PubMed

11. Branda JA, Body BA, Boyle J, Branson BM, Dattwyler RJ, Fikrig E, Gerald NJ, Gomes-Solecki M, Kintrup M, Ledizet M, Levin AE, Lewinski M, Liotta LA, Marques A, Mead PS, Mongodin EF, Pillai S, Rao P, Robinson WH, Roth KM, Schriefer ME, Slezak T, Snyder J, Steere AC, Witkowski J, Wong SJ, Schutzer SE. Steere AC21, Witkowski J, Wong SJ, Schutzer SE. Advances in serodiagnostic testing for Lyme disease are at hand. Clin Infect Dis 2018;66(7):1133-9. DOI PubMed

12. National Institute for Health and Care Excellence. Guidance: Lyme disease. London (UK): NICE; 2018 (Accédé 30-092019). https://www.nice.org.uk/guidance/ng95
13. Waddell LA, Greig J, Mascarenhas M, Harding S, Lindsay R, Ogden N. The accuracy of diagnostic tests for Lyme disease in humans, a systematic review and meta-analysis of North American research. PLoS One 2016;11(12):e0168613. DOl PubMed

14. Shapiro ED. Lyme disease. N Engl J Med 2014;371(7):684. DOl PubMed

15. Branda JA, Linskey K, Kim YA, Steere AC, Ferraro MJ. Two-tiered antibody testing for Lyme disease with use of 2 enzyme immunoassays, a whole-cell sonicate enzyme immunoassay followed by a VlsE C6 peptide enzyme immunoassay. Clin Infect Dis 2011;53(6):541-7. DOI PubMed

16. Molins CR, Delorey MJ, Sexton C, Schriefer ME. Lyme Borreliosis serology: Performance of several commonly used laboratory diagnostic tests and a large resource panel of well-characterized patient samples. J Clin Microbiol 2016;54(11):2726-34. DOI PubMed

17. Lipsett SC, Branda JA, McAdam AJ, Vernacchio L, Gordon CD, Gordon CR, Nigrovic LE. Evaluation of the C6 Lyme enzyme immunoassay for the diagnosis of Lyme disease in children and adolescents. Clin Infect Dis 2016;63(7):922-8. DOI PubMed

18. Branda JA, Strle K, Nigrovic LE, Lantos PM, Lepore TJ, Damle NS, Ferraro MJ, Steere AC. Evaluation of modified 2-tiered serodiagnostic testing algorithms for early Lyme disease. Clin Infect Dis 2017;64(8):1074-80. DOI PubMed

19. Pegalajar-Jurado A, Schriefer ME, Welch RJ, Couturier MR, MacKenzie T, Clark RJ, Ashton LV, Delorey MJ, Molins CR. Evaluation of modified two-tiered testing algorithms for Lyme disease laboratory diagnosis using well-characterized serum samples. J Clin Microbiol 2018;56(8):e01943-17. DOl PubMed

20. Lipsett SC, Branda JA, Nigrovic LE. Evaluation of the modified two-tiered testing (MTTT) method for the diagnosis of Lyme disease in children. J Clin Microbiol. 201957(10):e00547-19. DOl PubMed

21. Molins CR, Sexton C, Young JW, Ashton LV, Pappert R, Beard $\mathrm{CB}$, Schriefer ME. Collection and characterization of samples for establishment of a serum repository for lyme disease diagnostic test development and evaluation. J Clin Microbiol 2014;52(10):3755-62. DOI PubMed

22. Branda JA, Strle F, Strle K, Sikand N, Ferraro MJ, Steere AC. Performance of United States serologic assays in the diagnosis of Lyme borreliosis acquired in Europe. Clin Infect Dis 2013;57(3):333-40. DOI PubMed

23. U.S. Food and Drug Administration. FDA News Release: FDA clears new indications for existing Lyme disease tests that may help streamline diagnoses. FDA; 2019 (Accédé 21-09-2019). https://www.fda.gov/news-events/ press-announcements/fda-clears-new-indications-existin g-lyme-disease-tests-may-help-streamline-diagnoses 
24. Mead P, Petersen J, Hinckley A. Updated CDC recommendation for serologic diagnosis of Lyme disease. MMWR Morb Mortal Wkly Rep 2019;68(32):703. DOI PubMed

25. Zweitzig D, Kopnitsky M. and Zeus Scientific. Validation of a modified two-tiered testing (MTTT) algorithm for the improved diagnosis of Lyme disease. Unpublished Technical report; 2019. 13 pp.

26. Wormser GP, Levin A, Soman S, Adenikinju O, Longo MV, Branda JA. Comparative cost-effectiveness of two-tiered testing strategies for serodiagnosis of lyme disease with noncutaneous manifestations. J Clin Microbiol 2013;51(12):4045-9. DOl PubMed

27. Kalish RA, McHugh G, Granquist J, Shea B, Ruthazer $\mathrm{R}$, Steere AC. Persistence of immunoglobulin $\mathrm{M}$ or immunoglobulin $\mathrm{G}$ antibody responses to Borrelia burgdorferi 10-20 years after active Lyme disease. Clin Infect Dis 2001;33(6):780-5. DOI PubMed

28. Seriburi V, Ndukwe N, Chang Z, Cox ME, Wormser GP. High frequency of false positive IgM immunoblots for Borrelia burgdorferi in clinical practice. Clin Microbiol Infect 2012;18(12):1236-40. DOI PubMed

29. Fallon BA, Pavlicova M, Coffino SW, Brenner C. A comparison of lyme disease serologic test results from 4 laboratories in patients with persistent symptoms after antibiotic treatment. Clin Infect Dis 2014;59(12):1705-10. DOI PubMed

30. Lantos PM, Lipsett SC, Nigrovic LE. False positive Lyme disease IgM immunoblots in children. J Pediatr 2016;174:267-269.e1. DOI PubMed

31. Webber BJ, Burganowski RP, Colton L, Escobar JD, Pathak SR, Gambino-Shirley KJ. Lyme disease overdiagnosis in a large healthcare system: a population-based, retrospective study. Clin Microbiol Infect 2019;25(10):1233-8. DOI PubMed

32. Akin E, McHugh GL, Flavell RA, Fikrig E, Steere AC. The immunoglobulin (lgG) antibody response to OspA and OspB correlates with severe and prolonged Lyme arthritis and the IgG response to P35 correlates with mild and brief arthritis. Infect Immun 1999;67(1):173-81. DOl PubMed
33. Steere AC. Treatment of Lyme arthritis. J Rheumatol 2019;46(8):871-3. DOI PubMed

34. Davis I, McNeil SA, Allen W, MacKinnon-Cameron D, Wilson K, Bernat K, Dibernardo A, Lindsay LR, Hatchette TF. Performance of two EIA algorithm for Lyme disease (LD) in Nova Scotia. JAMMI. 2019;4(S1): poster P57. DOI

35. Gasmi S, Ogden NH, Lindsay LR, Burns S, Fleming $S$, Badcock J, Hanan S, Gaulin C, Leblanc MA, Russell C, Nelder M, Hobbs L, Graham-Derham S, Lachance L, Scott AN, Galanis E, Koffi JK. Surveillance de la maladie de Lyme au Canada, de 2009 à 2015. Relevé des maladies transmissibles au Canada 2017;43(10):219-25. DOI

36. Lantos PM, Branda JA, Boggan JC, Chudgar SM, Wilson EA, Ruffin F, Fowler V, Auwaerter PG, Nigrovic LE. Poor Positive predictive value of Lyme disease serologic testing in an area of low disease incidence. Clin Infect Dis 2015;61(9):1374-80. DOl PubMed

37. Tugwell $P$, Dennis DT, Weinstein A, Wells $G$, Shea B, Nichol G, Hayward R, Lightfoot R, Baker P, Steere AC. Laboratory evaluation in the diagnosis of Lyme disease. Ann Intern Med 1997;127(12):1109-23. DOI PubMed

38. Mechai S, Margos G, Feil EJ, Lindsay LR, Ogden NH. Complex population structure of Borrelia burgdorferi in southeastern and south central Canada as revealed by phylogeographic analysis. Appl Environ Microbiol 2015;81(4):1309-18. DOl PubMed

39. Ogden NH, Arsenault J, Hatchette TF, Mechai S, Lindsay LR. Antibody responses to Borrelia burgdorferi detected by western blot vary geographically in Canada. PLoS One 2017;12(2):e0171731. DOI PubMed 